\title{
Epidermal Growth Factor Receptor Mutations in Colorectal Cancer Patients
}

\author{
Bo-Young Oh, Ryung-Ah Lee, Soon-Sup Chung, Kwang Ho Kim \\ Department of Surgery, Ewha Womans University School of Medicine, Seoul, Korea
}

Purpose: The epidermal growth factor receptor (EGFR) plays an important role in tumorigenesis and tumor progression of colorectal cancer and leads to the activation of intracellular signaling pathways. The use of anti-EGFR-targeted therapy has increased for patients with colorectal cancer, but patients with EGFR mutations will be resistant to anti-EGFR-targeted therapy. The identification of gene mutations is critical in cancer treatment; therefore, the aim of this study is to investigate the incidences of EGFR mutations in colorectal cancer patients in Korea.

Methods: We retrospectively reviewed 58 colorectal cancer patients who underwent surgery between 2003 and 2006. We analyzed their EGFR mutations in four loci by DNA sequencing. In addition, we analyzed the correlation between the presence of EGFR mutation and patients' clinicopathologic features.

Results: Of the 58 patients, 35 patients were male and 23 were female. Their mean age was $63.28 \pm 11.18$ years. Two patients (3.45\%) were diagnosed as stage Tis, 7 patients (12.07\%) as stage I, 24 patients $(41.38 \%)$ as stage II, 20 patients (34.48\%) as stage III, and 5 patients $(8.62 \%)$ as stage IV. As a result of mutational analysis, EGFR mutations on exon 20 were detected in 13 patients (22.41\%, G $\rightarrow$ A transitions). No EGFR mutations were detected on exons 18,19 , and 21. EGFR mutation was increased in the earlier stage and in the absence of lymph node metastasis $(\mathrm{P}=0.028)$.

Conclusion: The incidence of EGFR mutation in Korean colorectal cancer patients is $22.41 \%$. In addition, EGFR mutation was significantly increased in the earlier stage and in the absence of lymph node metastasis.

\section{Keywords: Epidermal growth factor receptor; Mutation; Colorectal neoplasms}

\section{INTRODUCTION}

Colorectal cancer is one of the malignant tumors that are found most frequently around the world $[1,2]$, and it newly occurs in around 500 thousand patients every year [3], ranking third among causes of death for cancer patients in Western countries [1,3]. This tendency was also observed in South Korea. According to data from the Korea Central Cancer Registry published in 2008, the incidence and the mortality rates of colorectal cancer ranked third and fourth, respectively. Although about $70-80 \%$ at diagnosis can be cured

Received: March 30, 2011 Accepted: June 6, 2011

Correspondence to: Kwang Ho Kim, M.D.

Department of Surgery, Ewha Womans University School of Medicine,

911-1 Mok-dong, Yangcheon-gu, Seoul 158-710, Korea

Tel: +82-2-2650-5585, Fax: +82-2-2644-7984

E-mail: eastgate@ewha.ac.kr

(C) 2011 The Korean Society of Coloproctology

This is an open-access article distributed under the terms of the Creative Commons Attribution NonCommercial License (http://creativecommons.org/licenses/by-nc/3.0) which permits unrestricted noncommercial use, distribution, and reproduction in any medium, provided the original work is properly cited. completely through surgical treatment, approximately $50 \%$ of patients were reported to show metastasis $[1,4]$. Therefore, systemic chemotherapy, in addition to surgical treatment, has been widely applied to improve the survival rate, but existing chemotherapy combining oxaliplatin or irinotecan with 5-FU has not yet achieved a satisfactory survival rate for metastatic colorectal cancer patients $[1,4]$. Thus, to enhance the treatment outcome for patients with resistance to existing antitumor agents, new therapies have drawn attention, and targeted therapy is representative in these terms [4]. Many targeted agents have been being developed. In particular, agents targeted at signal transduction for cell growth have been actively studied, and antitumor agents targeted at epidermal growth factor receptor (EGFR) are representative [5]. EGFR is a cell-surface receptor for which expression increases in various malignant tumors, including colorectal cancer, and which affects cell growth and proliferation, metastasis, angiogenesis, and cell death through intracellular signal transduction $[4,5]$. These days some agents targeted at EGFR are being used, and they have contributed to improvements in the survival rate for colorectal cancer patients. However, patients with EGFR mutation have resistance to the therapy 
targeted at it, so checking for the existence of the mutation is helpful in deciding on an appropriate treatment. However, the incidence of the EGFR mutation in colorectal cancer patients has not yet been determined, and especially there are nearly no reports on the incidence rate for Koreans. Therefore, this study was conducted to investigate the incidence rate of the EGFR mutation in colorectal cancer patients in Korea and to examine the correlation between clinical and histopathologic characteristics and the existence of the mutation.

\section{METHODS}

This study investigated retrospectively 58 colorectal cancer patients surgically treated at the Surgery Department of Ewha Womans University Mokdong Hospital from January 2003 to December 2006. There were no limitations on the gender and the age of the subjects, but patients undergoing preoperative chemotherapy or radiotherapy were excluded from this study. After surgery, all subjects were diagnosed histopathologically as having a primary colon or rectal adenocarcinoma, and their specimens obtained during the surgery were analyzed.

To evaluate the mutation of EGFR, DNA was extracted from frozen sections of the specimens by using the QIAamp DNA Mini kit (Qiagen, Hilden, Germany) and following a conventional procedure [6]. Primers of exon 18 (codon 719), 19 (codon 746-750), 20 (codon 787), and 21 (codon 858) of the EGFR gene were utilized for amplification (Table 1). EGFR exons were amplified from $100 \mathrm{ng}$ of DNA through the polymerase chain reaction (PCR) in a $20 \mu \mathrm{L}$ mixed solution including $2 \mu \mathrm{L}$ of $10 \times$ buffer (Roche, Mannheim, Germany), 1.7-2.5 of mmol/ $\mathrm{L} \mathrm{MgCl}_{2}, 0.3 \mu \mathrm{M}$ of each EGFR primer pair (Roche), $250 \mu \mathrm{mol} / \mathrm{L}$ of deoxynucleotide triphosphates and 2.5 units of DNA polymerase (Roche). Amplifications involved a 5-minute initial denaturation step at $94^{\circ} \mathrm{C}$, followed by 30 cycles of 1 minute at $94^{\circ} \mathrm{C}, 1$ minute at $59^{\circ} \mathrm{C}$, and 1 minute at $72^{\circ} \mathrm{C}$, and a 10 -minute final extension step at $72^{\circ} \mathrm{C}$. All PCR products were separated with $2 \%$ agarose gel by using the QIAgen gel extraction kit (Qiagen), and they were used as templates for DNA

Table 1. Primers used for amplification and sequencing of epidermal growth factor receptor genes

\begin{tabular}{|c|c|}
\hline Amplified fragment & Primer sequence \\
\hline \multirow[t]{2}{*}{ Exon 18} & F: 5'-TCCAAATGAGCTGGCAAGTG \\
\hline & R: 5'-TCCCAAACACTCAGTGAAACAAA \\
\hline \multirow[t]{2}{*}{ Exon 19} & F: 5'-ATGTGGCACCATCTCACAATTGCC \\
\hline & R: 5'-CCACACAGCAAAGCAGAAACTCAC \\
\hline \multirow[t]{2}{*}{ Exon 20} & F: 5'-CATTCATGCGTCTTCACCTG \\
\hline & R: 5'-CATATCCCCATGGCAAACTC \\
\hline \multirow[t]{2}{*}{ Exon 21} & F: 5'-GCTCAGAGCCTGGCATGAA \\
\hline & R: 5'-CATCCTCCCCTGCATGTGT \\
\hline
\end{tabular}

F, forward; R, reverse. sequencing. The sequencing was conducted by repeating 25 cycles of 10 -second, 5-second and 4-minute treatments at $96^{\circ} \mathrm{C}, 50^{\circ} \mathrm{C}$, and $60^{\circ} \mathrm{C}$, respectively, with $20 \mathrm{ng}$ of each separated PCR product in a $20 \mu \mathrm{L}$ mixed solution for sequencing, including $8 \mu \mathrm{L}$ of Big Dye Terminator v3.1 (Applied Biosystem, Foster City, CA, USA) and $0.1 \mu \mathrm{mol} / \mathrm{L}$ of PCR primer, by using an ABI PRISM 3100 DNA Analyzer (Applied Biosystem). The data were analyzed with Sequencer 3.1.1 software (Applied Biosystems) to compare sequence variations. In addition, clinical characteristics, such as gender, age, tumor markers, survival and histopathologic characteristics, like the location, the stage and the differentiation of lesions and the existence of lymph node metastasis, were examined based on the medical records, and the correlations between these characteristics and the EGFR mutation were investigated.

The correlations between the clinical and the histopathologic characteristics of the patients and the mutation were analyzed with

Table 2. Patient's clinicopathologic features

\begin{tabular}{|c|c|}
\hline Variable & №. (\%) \\
\hline Total & 58 \\
\hline \multicolumn{2}{|l|}{ Gender } \\
\hline Male & $35(60.34)$ \\
\hline Female & 23 (39.66) \\
\hline Mean age (yr) & $63.28 \pm 11.18$ \\
\hline \multicolumn{2}{|l|}{ CEA (ng/mL) } \\
\hline$\leq 5$ & 41 (70.69) \\
\hline$>5$ & $17(29.31)$ \\
\hline \multicolumn{2}{|c|}{ CA19-9 (U/mL) } \\
\hline$\leq 27$ & $51(87.93)$ \\
\hline$>27$ & 7 (12.07) \\
\hline \multicolumn{2}{|l|}{ Location } \\
\hline Colon & 37 (63.79) \\
\hline Rectum & 21 (36.21) \\
\hline \multicolumn{2}{|l|}{ Differentiation } \\
\hline Well & 8 (13.79) \\
\hline Moderate & 45 (77.59) \\
\hline Poor & $5(8.62)$ \\
\hline \multicolumn{2}{|l|}{ LN metastasis } \\
\hline Absent & $33(56.90)$ \\
\hline Present & $25(43.10)$ \\
\hline \multicolumn{2}{|l|}{ Stage } \\
\hline Tis & $2(3.45)$ \\
\hline I & 7 (12.07) \\
\hline$\|$ & $24(41.38)$ \\
\hline III & $20(34.48)$ \\
\hline IV & $5(8.62)$ \\
\hline
\end{tabular}

LN, lymph node; Tis, tumor in situ. 
the Person's the Fisher's exact test and linear-by-linear association. After dividing the subjects into mutant-type and wild-type groups, we investigated the overall survival and the disease-free survival of each group by using the Kaplan-Meier method, and the survivals of the two groups were compared through a univariate analysis using the log-rank test. The program used for the analysis was SPSS ver. 12.0 (SPSS Inc., Chicago, IL, USA), and a $\mathrm{P}$-value of less than 0.05 was considered to be significant.

\section{RESULTS}

\section{Clinical and histopathologic characteristics of the subjects}

Totally, there were 58 subjects, consisting of 35 males $(60.34 \%)$ and 23 females (39.66\%), and their mean age was $63.28 \pm 11.18$ years (range, 36 to 84 years). Preoperative CEA and CA19-9 levels were higher than the normal ranges in 17 (29.31\%) and 7 (12.07\%) subjects, respectively (Table 2).

All of the subjects were surgically treated due to colorectal cancer, and after the surgery, they were diagnosed histopathologically as having a colon or a rectal adenocarcinoma. The lesions were located on the colon or the rectum in 37 (63.79\%) and 21 (36.21\%) patients, respectively. For histopathologic cell differentiation, 8 (13.79\%), 45 (77.59\%), and 5 (8.62\%) cases were well differentiated, moderately differentiated and poorly differentiated, respectively. Lymph node metastasis was found in 25 (43.10\%) cases. Metastasis to other organs at diagnosis was occurred in 5 cases, and all of them showed metastasis to the liver. In addition, the recorded histopathologic stages were Tis and stage 1, 2, 3, and 4 in 2 (3.45\%), 7 (12.07\%), 24 (41.38\%), 20 (34.48\%), and 5 (8.62\%) cases, respectively (Table 2 ).

\section{Incidence rate of the EGFR mutation}

The EGFR mutations on exons 18 (codon 719), 19 (codon 746750), 20 (codon 787), and 21 (codon 858) of all specimens were analyzed through sequencing. According to the results, EGFR mu-

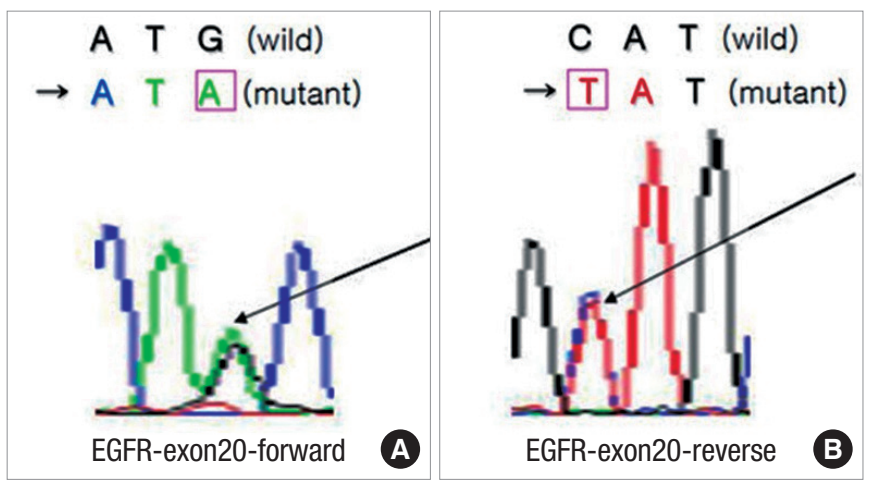

Fig. 1. Sequencing results for the epithelial growth factor receptor (EGFR) mutation. Partial nucleotide sequences of the wild and the mutant in exon 20 of the EGFR gene. (A) Forward: transition of G to A (arrow), leading to the substitution of methionine for isoleucine and (B) Reverse: transition of C to $\mathrm{T}$ (arrow). tations were observed on exon 20 in $13(22.41 \%)$ patients. They were point mutations from ATG to ATA and produced amino acid substitution from methionine to isoleucine (Fig. 1). However, no mutations were found on exons 18,19 , and 21.

\section{Correlation between the clinical and histopathologic characteristics and the EGFR mutation}

Correlations between the clinical and histopathologic characteristics and the EGFR mutation were investigated. When the existence of the EGFR mutation was assessed by classifying the stage into Tis-stage 2 and stage 3-4 groups, the mutations were found in 11 out of $33(56.90 \%)$ patients with colorectal cancer at Tis-stage 2 and in 2 out of $25(43.10 \%)$ patients with colorectal cancer at stage 3-4. In the comparison between the two groups, the earlier stage group showed a significantly higher incidence rate of EGFR mutation $(\mathrm{P}=0.028)$.

Table 3. Relationship between the EGFR mutation and the clinicopathologic features

\begin{tabular}{|c|c|c|c|}
\hline Variable & Number & $\begin{array}{c}\text { EGFR } \\
\text { mutation (\%) }\end{array}$ & P-value \\
\hline Age (yr) & & & 0.74 \\
\hline$<60$ & 19 & 5 (26.32) & \\
\hline$\geq 60$ & 39 & 8 (20.51) & \\
\hline Gender & & & 0.21 \\
\hline Male & 35 & $10(28.57)$ & \\
\hline Female & 23 & $3(13.04)$ & \\
\hline CEA (ng/mL) & & & 1 \\
\hline $5 \leq$ & 41 & 9 (21.95) & \\
\hline$>5$ & 17 & $4(23.53)$ & \\
\hline CA19-9 (U/mL) & & & 1 \\
\hline $27 \leq$ & 51 & $12(23.53)$ & \\
\hline$>27$ & 7 & $1(14.29)$ & \\
\hline Stage & & & 0.028 \\
\hline Tis-II & 33 & 11 (33.33) & \\
\hline III-IV & 25 & $2(8.00)$ & \\
\hline Location & & & 0.751 \\
\hline Colon & 37 & 9 (24.32) & \\
\hline Rectum & 21 & $4(19.05)$ & \\
\hline Differentiation & & & 0.267 \\
\hline Well & 8 & $0(0)$ & \\
\hline Moderate & 45 & $12(26.67)$ & \\
\hline Poor & 5 & $1(20.00)$ & \\
\hline LN metastasis & & & 0.028 \\
\hline Absent & 33 & 11 (33.33) & \\
\hline Present & 25 & $2(8.00)$ & \\
\hline
\end{tabular}

EGFR, epithelial growth factor receptor; Tis, tumor in situ; LN, lymph node. 

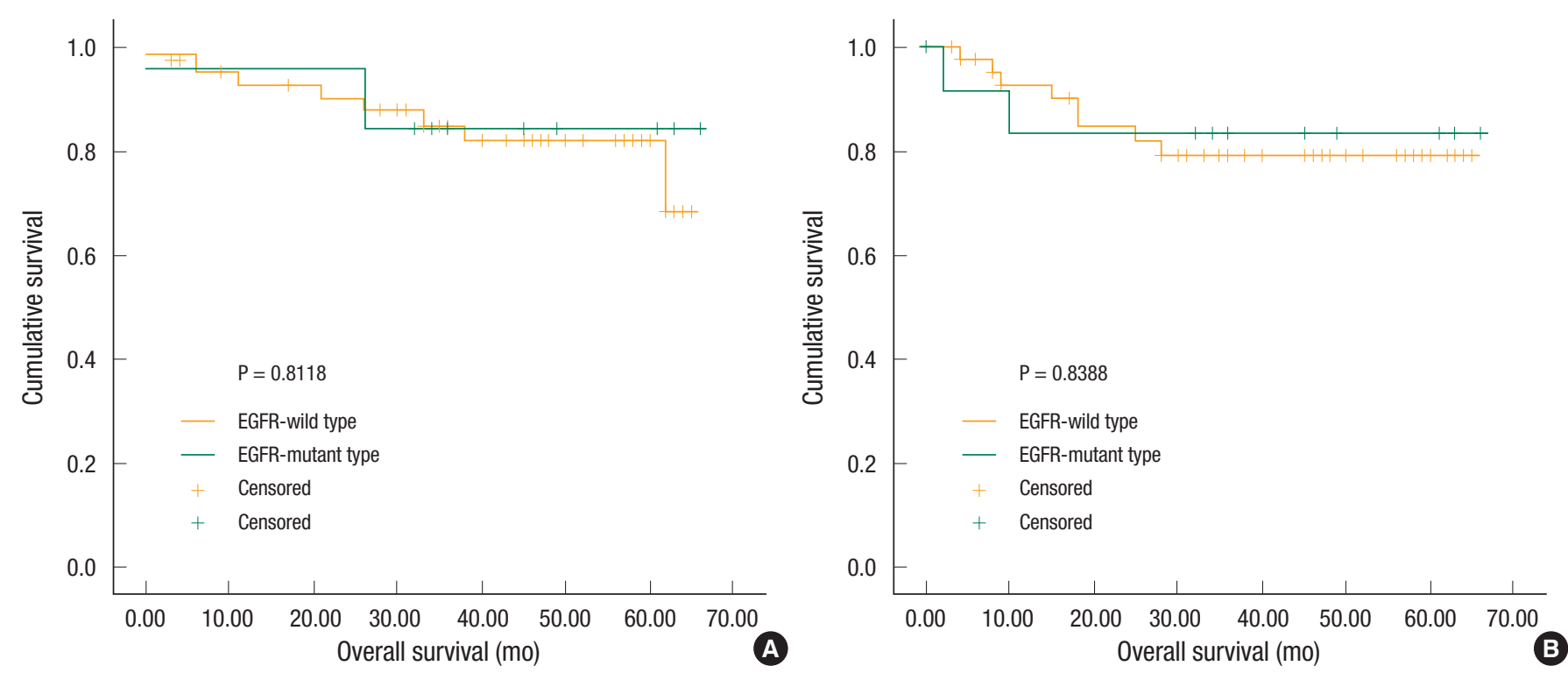

Fig. 2. Overall survival and disease-free survival according to epithelial growth factor receptor (EGFR) status. (A) There was no significant difference between the EGFR-wild group and the EGFR-mutant group as to overall survival $(\mathrm{P}=0.8118)$. (B) EGFR mutational status was not associated with any significant influence on disease-free survival $(P=0.8388)$.

The subjects were also divided based on lymph node metastasis. Patients with and without metastasis were 25 (43.10\%) and 33 (56.90\%), respectively, and EGFR mutations were found in 2 and 11 cases, respectively. Therefore, patients without lymph node metastasis recorded a significantly higher incidence rate for the mutation ( $\mathrm{P}=0.028)$. However, gender, age, CEA and CA19-9 levels, and the location and the differentiation of lesions were not significantly related to the existence of the EGFR mutation (Table 3).

Overall survival and disease-free survival were analyzed by classifying the subjects into two groups based on the existence of the EGFR mutation. The number of patients with the EGFR mutation was 13 (22.41\%), and their average overall survival and disease-free survival were $57.85 \pm 5.49$ and $56.00 \pm 6.47$ months, respectively. The number of patients without the mutation was 45 (77.59\%), and their overall survival and disease-free survival were $56.66 \pm 2.79$ and $54.83 \pm 3.24$ months, respectively. When the overall survival and disease-free survival of the two groups were compared, there was no significant difference due to the EGFR mutation (Fig. 2).

\section{DISCUSSION}

EGFR, called as ErbB1, is a member of the ErbB family [7], and it is a transmembrane glycoprotein that forms part of the tyrosine kinase receptor protein. Its size is $170 \mathrm{kD}$, and it consists of 1186 amino acid polypeptide chains [8]. The ErbB family is tyrosine kinase receptors activated by different kinds of ligands, and it consists of ErbB1 (EGFR, HER1), ErbB2 (HER2), ErbB3 (HER3), and ErbB4 (HER4). Each of them has extracellular, transmembrane and intracellular domains [4]. Their major ligands are EGF, TGF- $\alpha$, amphiregulin and betacellulin [1]. If these ligands combine with domains, main intracellular signal transduction, such as the Ras/ Raf/MAPK pathway and the PI3K/Akt pathway, are activated through dimerization of receptors and continuous autophosphorylation and influence the differentiation and the metastasis of tumors, angiogenesis and cell death $[1,9]$.

The expression of EGFR is known to be increased for many malignant tumors, like head and neck cancer, stomach cancer, colorectal cancer, prostate cancer, pancreatic cancer and lung cancer [10], and its over-expression was found in $65-75 \%$ of patients with advanced colorectal cancer [1]. Although many studies on the correlation between the expression rate of EGFR and the prognosis for patients have been performed, no correlation has been determined. While for head and neck cancer, ovarian cancer, bladder cancer and esophageal cancer, its expression has been reported to be related to the survival rate of patients, for colorectal cancer, the correlation is not clear. According to most reports, the expression of EGFR in colorectal cancer patients is not related to clinical factors such as gender, age, the location and the differentiation of tumors, and lymph node metastasis [11]. However, some studies insist that the expression is associated with progression, differentiation, lymph node metastasis and recurrence of colorectal cancer [5]. Therapies targeted at EGFR can be largely divided into two types: monoclonal antibodies blocking the interaction between ligands and extracellular domains and tyrosine kinase inhibitors hindering intracellular signal transduction pathways [1]. Monoclonal antibodies currently used are cetuximab and panitumumab, and they are reported to be effective in monotherapy or in combination therapy for colorectal cancer patients with resistance to irinotecan or oxaliplatin [1]. In addition, tyrosine kinase inhibitors are gefitinib and erlotinib, and they are applied mainly for small-cell 
lung carcinomas and are known to be ineffective in colorectal cancer patients [1].

Common antitumor agents used for colorectal cancer are 5-FU, oxaliplatin and irinotecan. As signal transduction pathways caused by EGFR and K-ras are known to be related to the pathogenesis of colorectal cancer, a therapy targeted at EGFR is applied when there is resistance to existing antitumor agents and EGFR is expressed. However, some studies reporting resistance to these agents [12] suggested that the resistance was associated with a mutation of EGFR, so efforts have been made to investigate the incidence of patients with the mutation and to develop therapies fitting them.

Most of studies on EGFR mutation have been conducted with small-cell lung carcinomas, and the mutation was reported to be observed in $45 \%$ of the cases [13]. According to studies by regions, the EGFR mutation was found in $28 \%$ of small-cell lung carcinoma patients in Greece [14], and the rate of the mutation was 15\% in Korea [15]. Another study published in Korea reported a higher incidence rate of $24 \%$ and revealed no significant correlation of the EGFR mutation with clinicopathologic characteristics, including age, gender, stage and differentiation [6]. Unlike small-cell lung carcinomas, for which a correlation with the EGFR mutation has been reported in many studies, for colorectal cancer, only the incidence rate of the mutation has been reported, and there has been nearly no study on its correlation with clinicopathologic characteristics $[13,16]$. Some research published in Western countries found that the incidence of the EGFR mutation in colorectal cancer patients was very low. Barber et al. [16] reported that it was $0.34 \%$ of 293 patients, and Ogino et al. [17] reported that the mutation on exon 18 was observed in one (3.3\%) of 30 patients. Meanwhile, studies in Japan showed slightly higher incidence rates compared to Western countries. Nagahara et al. [13] revealed that EGFR mutations were found in four (12\%) of 33 colorectal cancer patients and that the mutations had occurred on exon 19 in 2 patients and on exon 20 in 2 patients. Our study investigated the incidence rate of the EGFR mutation for colorectal cancer patients in Korea and found that 13 out of 58 (22.4\%) had the mutation on exon 20 . The patients at earlier stage and with no lymph node metastasis tended to show a significantly higher incidence rate of the mutation, but the overall survival and the disease-free survival were not significantly different. The correlations of the mutation with clinicopathologic characteristics, such as gender, age, CEA and CA19-9 levels, and the location and the differentiation of lesions, were also investigated, but no significant correlations were found.

This study had some limitations. The sample size was small, and the data were analyzed retrospectively. The subjects were patients in a single medical institution, so the results may not apply to all colorectal cancer patients. Because the subjects did not undergo the same treatments, the correlation between the EGFR mutation and clinical outcomes could not be evaluated properly. However, considering that there have been no studies on the EGFR mutation in colorectal cancer patients, our study is meaningful as it investigates the incidence rate of the mutation.
The incidence rate of the EGFR mutation in colorectal cancer patients in Korea was $22.41 \%$, and it was significantly higher in the patients at earlier stage and without lymph node metastasis. If a study with a larger population is conducted in the future, it will produce more meaningful outcomes. In addition, examination to check the EGFR mutation before targeted therapy for colorectal cancer patients is necessary.

\section{CONFLICT OF INTEREST}

No potential conflict of interest relevant to this article was reported.

\section{ACKNOWLEDGMENTS}

This was presented at the 2010 Fall Conference of the Korea Society of Clinical Oncology and the 12th China-Japan-Korea Colorectal Cancer Symposium.

\section{REFERENCES}

1. de Castro-Carpeno J, Belda-Iniesta C, Casado Saenz E, Hernandez Agudo E, Feliu Batlle J, Gonzalez Baron M. EGFR and colon cancer: a clinical view. Clin Transl Oncol 2008;10:6-13.

2. Ponz-Sarvise M, Rodriguez J, Viudez A, Chopitea A, Calvo A, Garcia-Foncillas J, et al. Epidermal growth factor receptor inhibitors in colorectal cancer treatment: what's new? World J Gastroenterol 2007;13:5877-87.

3. Sameer AS, Chowdhri NA, Abdullah S, Shah ZA, Siddiqi MA. Mutation pattern of K-ras gene in colorectal cancer patients of Kashmir: a report. Indian J Cancer 2009;46:219-25.

4. Yun SH. Molecular targeted therapy in colorectal cancer. J Korean Soc Coloproctol 2004;20:180-8.

5. Kim HA, Lee RA, Hwang DY, Park SH. The significances of EGFR overexpression in colorectal cancer. J Korean Soc Coloproctol 2005;21:36-41.

6. Jang TW, Oak CH, Chang HK, Suo SJ, Jung MH. EGFR and KRAS mutations in patients with adenocarcinoma of the lung. Korean J Intern Med 2009;24:48-54.

7. Hynes NE, Lane HA. ERBB receptors and cancer: the complexity of targeted inhibitors. Nat Rev Cancer 2005;5:341-54.

8. Carpenter G, Cohen S. Epidermal growth factor. J Biol Chem 1990; 265:7709-12.

9. Zhou Y, Li S, Hu YP, Wang J, Hauser J, Conway AN, et al. Blockade of EGFR and ErbB2 by the novel dual EGFR and ErbB2 tyrosine kinase inhibitor GW572016 sensitizes human colon carcinoma GEO cells to apoptosis. Cancer Res 2006;66:404-11.

10. Kim ES, Khuri FR, Herbst RS. Epidermal growth factor receptor biology (IMC-C225). Curr Opin Oncol 2001;13:506-13.

11. McKay JA, Murray LJ, Curran S, Ross VG, Clark C, Murray GI, et al. Evaluation of the epidermal growth factor receptor (EGFR) in colorectal tumours and lymph node metastases. Eur J Cancer 2002; 38:2258-64. 


\section{Coloproctology Bo-Young Oh, et al.}

12. Di Fiore F, Blanchard F, Charbonnier F, Le Pessot F, Lamy A, Galais MP, et al. Clinical relevance of KRAS mutation detection in metastatic colorectal cancer treated by Cetuximab plus chemotherapy. Br J Cancer 2007;96:1166-9.

13. Nagahara H, Mimori K, Ohta M, Utsunomiya T, Inoue H, Barnard GF, et al. Somatic mutations of epidermal growth factor receptor in colorectal carcinoma. Clin Cancer Res 2005;11:1368-71.

14. Kalikaki A, Koutsopoulos A, Trypaki M, Souglakos J, Stathopoulos E, Georgoulias V, et al. Comparison of EGFR and K-RAS gene status between primary tumours and corresponding metastases in NSCLC. Br J Cancer 2008;99:923-9.
15. Park SH, Ha SY, Lee JI, Lee H, Sim H, Kim YS, et al. Epidermal growth factor receptor mutations and the clinical outcome in male smokers with squamous cell carcinoma of lung. J Korean Med Sci 2009;24:448-52.

16. Barber TD, Vogelstein B, Kinzler KW, Velculescu VE. Somatic mutations of EGFR in colorectal cancers and glioblastomas. N Engl J Med 2004;351:2883.

17. Ogino S, Meyerhardt JA, Cantor M, Brahmandam M, Clark JW, Namgyal C, et al. Molecular alterations in tumors and response to combination chemotherapy with gefitinib for advanced colorectal cancer. Clin Cancer Res 2005;11:6650-6. 